\title{
Application of conformal map theory for design of 2D ultrasonic array structure for NDT imaging application: a feasibility study
}

\author{
S. N. Ramadas ${ }^{1 *}$, J. C. Jackson ${ }^{2 *}$, J. Dziewierz ${ }^{2}$, R. O'Leary ${ }^{2}$, and A. Gachagan ${ }^{2}$ \\ ${ }^{1}$ Elster-Instromet NV/SA, Rijkmakerlaan 9, B-2910 Essen, Belgium \\ ${ }^{2}$ Centre for Ultrasonic Engineering, University of Strathclyde, Glasgow, UK
}

\begin{abstract}
Dimensional ultrasonic phased arrays are becoming increasingly popular in non-destructive evaluation (NDE). Sparse array element configurations are required to fully exploit the potential benefits of $2 \mathrm{D}$ phased arrays. This paper applies conformal mapping technique as a means of designing sparse 2D array layouts for NDE applications. Modelling using both Huygens' Field prediction theory and 2D Fast Fourier Transformation is employed to study the resulting new structure. A conformal power map was used that, for fixed beam width, was shown in simulations to have a greater contrast than rectangular or random arrays. A prototype aperiodic 2D array configuration, with operational frequency $\sim 3 \mathrm{MHz}$, for direct contact operation in steel was designed employing the array design principle described in this paper. Experimental results demonstrate a working sparse-array transducer capable of performing volumetric imaging.
\end{abstract}

Index Terms - 2-Dimensional, Ultrasonic, Phased Array, Imaging, Aperiodic Array, Conformal map, NDE

\section{INTRODUCTION}

U LTRASONIC phased arrays are becoming more popular in Non Destructive Evaluation (NDE) applications largely due to their flexible operational nature, which can result in faster inspection times [1,2]. The most recent developments have been in the design and implementation of 2-Dimensional (2D) array element configurations, which enables full volumetric inspection of a component [2-7]. There are a number of possible array layout configurations appropriate for use in NDE. Importantly, instead of a standard 2D array transducer, which consists of rectangular grid of elements, sparse and non-regular element configurations provide scope for reducing inter element crosstalk and more importantly could minimize manufacturing complexity [2- 4]. Such design configurations are also capable of producing radiation patterns that are suitable for high resolution imaging applications, often at the cost of imaging performance resulting from higher side lobe levels [3]. Consequently, careful design of a non-conventional array transducer for a specific requirement is essential in order to achieve optimum system performance.

This paper applies conformal mapping technique as means of designing sparse $2 \mathrm{D}$ array layouts. A conformal map is an angle preserving transformation, which has been widely used in many applications including aerofoils, fractals, and perfect lens design [8-10]. While there are a variety of interesting mapping functions available to exploit, this preliminary investigation presented is restricted to an fractional power transformation of the classical fully populated matrix 2D layout.

Extensive modelling using both Huygens' Field prediction theory and 2D Fast Fourier Transformation is employed to study performance of the resulting new array structure. A perturbation factor that governs the conformal transformation function allows further tuning of the resulting array design to suit a specific imaging application requirement. A comparison analysis against other common array designs, including densely populated rectangular and random 2D array configurations, is also presented. A prototype 2D sparse array, with $z^{4 / 3}$ conformal structure chosen for its interesting properties, was designed for direct contact operation in steel at an operational frequency $\sim 3 \mathrm{MHz}$, employing the conformal mapping methods described in this paper. First, Section II provides the necessary background by highlighting the key challenges involved in $2 \mathrm{D}$ array design. Section III introduces conformal mapping theory and how it is applied in sparse 2D array design methodology and finally prototype manufacture details, experiment evaluation and some concluding remarks are presented in Sections IV-VI.

${ }^{*}$ S.N. Ramadas and J.C. Jackson contributed equally to this article. 


\section{2D ULTRASONIC ARRAY DESIGN AND CHALLENGES}

An array transducer is simply a sensor comprising a number of individual piezoelectric elements (typically from 16 to 256 for NDE applications - a limit typically imposed by practical costs associated with the control of arrays with more elements) in a single housing. In other words, array transducers are a cluster of miniature piezoelectric elements and each element is wired and addressed individually via an array controller, to produce the desired ultrasound wavefront characteristic. Array transducers offer significant technical advantages over conventional single-probe ultrasonic inspections; mainly:

- Electronic scanning using a sub-aperture of an array

- Electronic beam steering - also referred to as S-scans

- Dynamic focusing in receive mode - one device can focus at several depths through a sample, leading to faster inspection of the volume of thick samples, and improved signal-to-noise ratio

However, all these technical advantages mentioned above come at the price of manufacturing complexity and cost. Even while considering a simple 1D linear array configuration, the array elements should be spaced no further than half wavelength distance in order to avoid any grating lobes (a form of aliasing caused by the periodic spacing of the array elements) [1]. At the same time, a wide spatial extension is required to achieve high resolution (as the lateral resolution of an array transducer is directly dependent on the array aperture size). Satisfying these two key requirements is costly and often prohibitive in many array configurations. Fig. 1 shows a simulation, using Huygens' principle, illustrating the effect of the unfocussed array beamwidth as a function of array aperture size. The obvious conclusion from Fig. 1 would be to increase the array aperture (with a concomitant increase in array elements to maintain pitch) to enhance the system resolution. Unfortunately, there is typically a limit on the array element count due to the cost and complexity of phased array controller instrumentation. Alternatively, increasing the element spacing in an array would reduce the element count. However, it introduces undesirable grating lobes when the element pitch is greater than $\lambda / 2$, as illustrated in the $1 \mathrm{D}$ array field directivity simulation shown in Fig. 2 . In this simulation, the array has been configured to focus at a steering angle of -30 degrees, with an additional grating lobe visible at 30 degrees in the lambda spacing configuration.

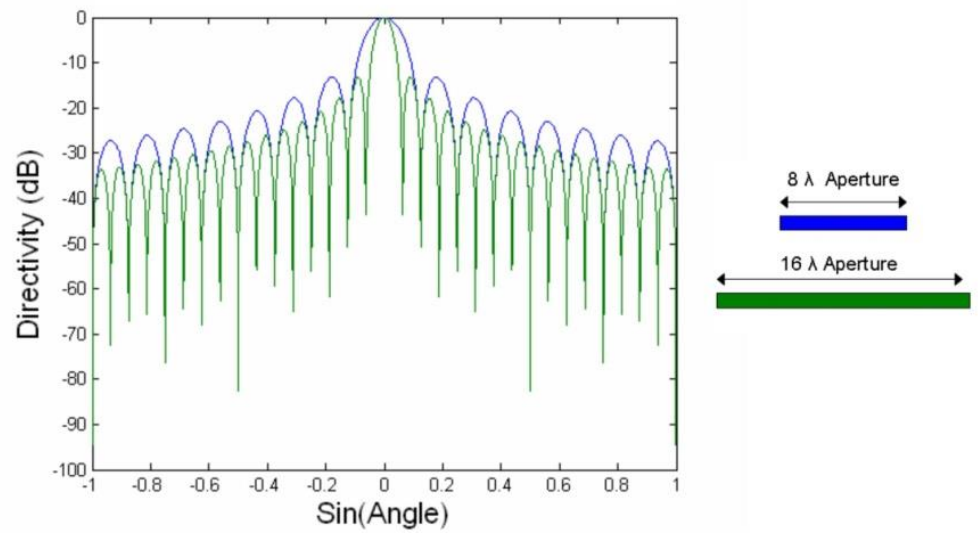

Fig. 1. Simulation illustrating the effect of array aperture on the unfocussed, unsteered, beam in the far field. Increasing the aperture decreases the beamwidth, thus increasing resolution, and increases the number of active elements, reducing the sidelobe levels. The ripples are sidelobes.

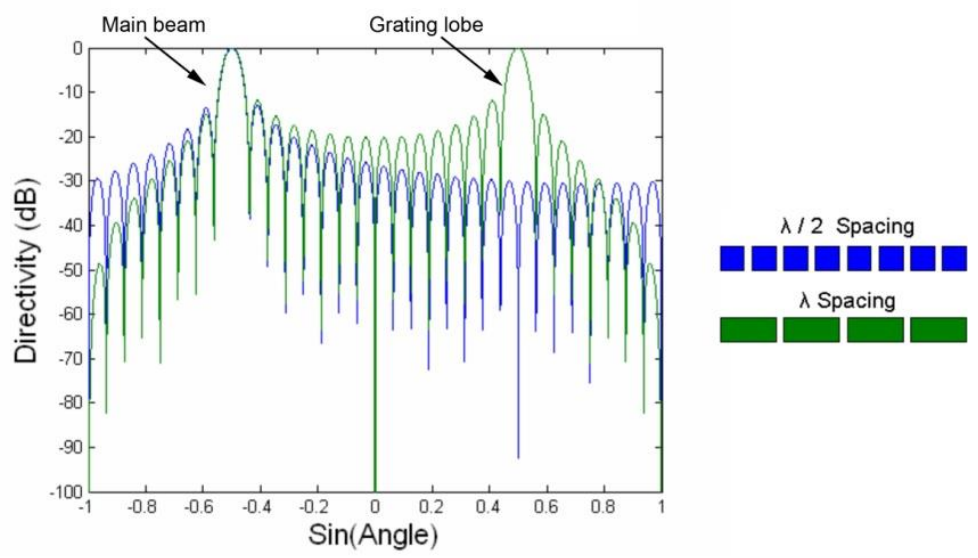

Fig. 2. Simulation illustrating the introduction of grating lobes in the output of a $1 \mathrm{D}$ array when the array spacing exceeds $\lambda / 2$. 
The main lobe, unfocussed, is steered to -30 degrees. A grating lobe appears at 30 degrees for element spacing of $\lambda$.

One of the most promising approaches to reducing the number of array elements is to make the array structure non periodic[11, 12], wherein a fully sampled array is thinned by removing a fraction of the original set of elements, thus obtaining a sparse array element layout. Alternatively, a random array layout could be generated using general purpose optimisation tools with specific constrain placed to avoid element overlap [3,5]. In both the cases, because of the non periodic nature of the array layout, aliasing effects are not present in such sparse array configurations. The main drawback however, is a potentially unacceptable high level of the side lobes present in the sparse array beam profile.

\section{CONFORMALLY MAPPED APERIODIC ARRAY DESIGN}

\section{A. Introduction to conformal mapping technique}

A conformal map is a transformation that preserves local angles, but not necessarily global shape, and is often used in the complex plane as a tool to convert a non-trivial geometry that would be difficult to manage analytically, into a more easily managed geometry. Uses for conformal mapping include the anaylsis of aerofoils through the famous Joukowksi transform [9], and more recently, the design of perfect metamaterial lenses and invisibility cloaks in the field known as transformation optics [10]. If a 2D surface can be parameterised by its coordinates $(x, y)$, then it is possible to create a complex coordinate $z=x+i y$ that does the same. This complex surface can be transformed by some function $w=f(z)$, such that $w=u+i v$ and $(u, v)$ are the new coordinates in the transformed space. The function $f(z)$ is conformal everywhere where its derivative $d f(z) / d z \neq 0$. Conformal maps have interesting mathematical properties, most notably that if a function satisfies Laplace's equation (i.e. it is harmonic) then a conformal map of this function will also satisfy Laplace's equation. Conformal maps are therefore useful for the analysis of physical problems governed by potentials. In designing aperiodic 2D arrays for ultrasonic transducers, a conformal map is a convenient and effective way to perturb a rectangular array into an aperiodic one, in a parametric fashion. Although conformal mapping is meant to be invertible, we do not use this property here and instead cast the transformed result back to Cartesian space.

\section{B. Conformal mapped aperiodic ultrasonic array structure}

Novel aperiodic arrays can be designed by beginning with a standard 2D rectangular array and perturbing the element positions. The goal is twofold - to create arrays with a large aperture that would not suffer from grating lobes, to reduce interelement cross-talk, and unwanted vibration mode selection. All of these problems can be attributed to the periodicity of the elements in a standard array. The conformal mapping process will be exemplified by considering a $2.5 \mathrm{MHz}, 121$ element conventional rectangular array design for direct operation into steel. Fig. 3(a) depicts the 11 by 11 rectangular grid layout, with $1.2 \mathrm{~mm}$ element pitch $(\lambda / 2)$, associated with this configuration and such a design is considered to represent a good balance between imaging performance and manufacturability. Each array element coordinate $\left(x_{m}, y_{m}\right)$ can be represented by a complex number $z_{m}=x_{m}+i y_{m}$. The range of this rectangular array can be normalised on the complex plane such that $|x| \leq 1,|y| \leq 1$. As an example of perturbing the array structure, the following will focus on a simple transformation, namely $w=z^{n}$, where $n$ is a real number. After the transformation, new coordinates $\left(u_{m}, v_{m}\right)$ can be extracted from $w_{m}=u_{m}+i v_{m}$ and scaled to create the desired overall aperture. The acoustic field produced from the array elements at position $\left(u_{m}, v_{m}\right)$ can be monitored by using Huygens' principle as shown in Fig. 3(b) or, in the far field, the 2D Fourier transform of the aperture (the Fraunhofer approximation). The Huygens simulation was calculated in MATLAB assuming a steel medium, and the Fraunhofer approximation in LabVIEW, scaled arbitrarily. Fig. 3(b) shows the structure of the beam pattern for a rectangular array, with periodic sidelobes and grating lobes orthogonal to the array pattern. Reduction, or ideally removal, of these lobes is desirable. 


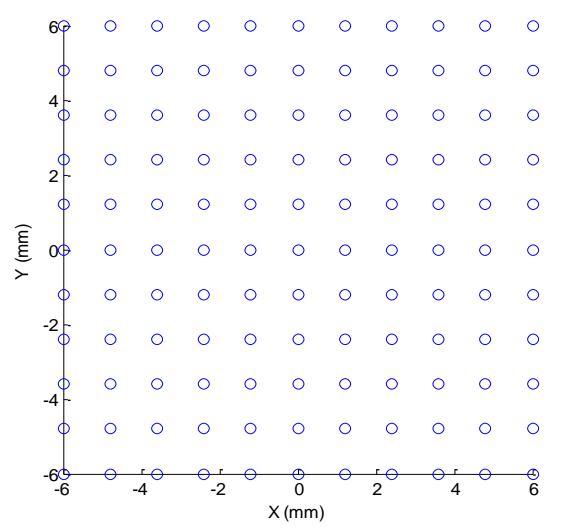

3(a) Array element layout - for simulation purposes, elements are assumed to be point sources

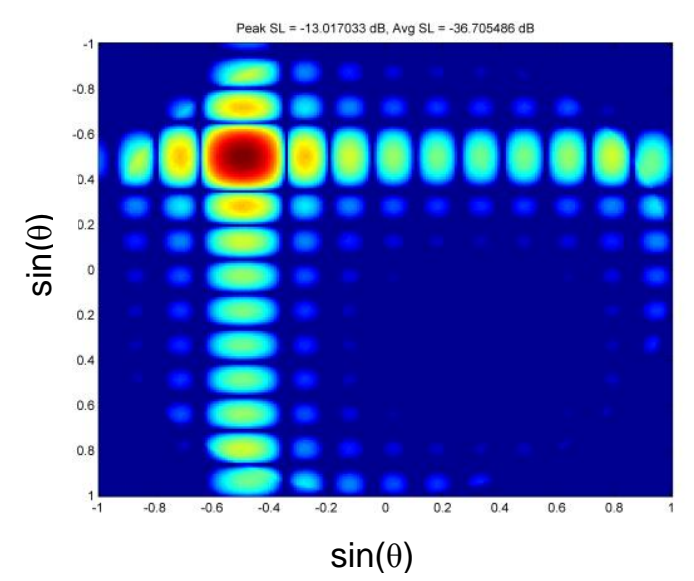

3(b) the steered directivity profile as a function of angle $\theta$ (steered to $30^{\circ}$ in the $\mathrm{x}$ and $\mathrm{y}$ directions) of the array determined using Huygens' principle showing the main and the sidelobes

Fig. 3. Standard 2.5MHz rectangular array configuration, and its directivity profile, used for comparison with sparse array designs.

Starting with $n=1$ and increasing its value, a variety of interesting choices emerge. For the $w=z^{n}$ transformation, the most interesting possible array designs occur in the bound $1<|n|<3$ : values of $n$ outside this range tend to create aperiodic arrays with a concentration of elements near the centre, or grossly asymmetric arrays. There are two noticeable advantages to this conformal perturbation.

First, the effect of small increases in the value of $n$ can quickly smear out and reduce grating lobes, as the rectangular grid layout becomes more distorted. Fig. 4 illustrates the effect of varying the power factor $n$, slightly. In this Fraunhofer simulation, the array element separation is kept larger than $\lambda / 2$ and the array is simulated to steer to an angle of 45 degrees in both the azimuthal and the elevation direction. Therefore, the desired focal point is identifiable in the top left hand corner of each image in Fig. 4. This simulation helps to illustrate clearly the presence of grating lobes in the case of a periodic array layout, when $n=1$. As shown in the other simulation images presented in Fig. 4, when the variable $n$ is varied slightly, the array periodicity starts to break. In other words, the peak grating lobe energy smears quickly, reducing grating lobe intensity. This also has the undesirable side-effect of bringing the overall side lobe level up, and has to be considered carefully as it affects directly the overall contrast of the image captured. Fig. 5 illustrates this point by plotting the peak and average sidelobe level when $1<n<1.5$.

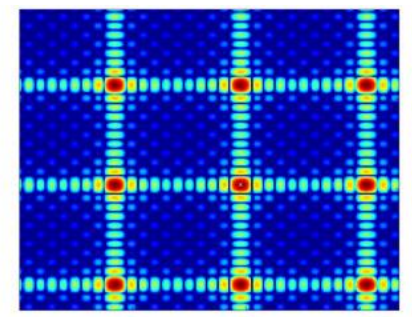

$n=1$

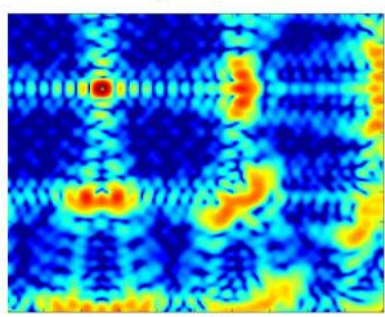

$n=1.06$

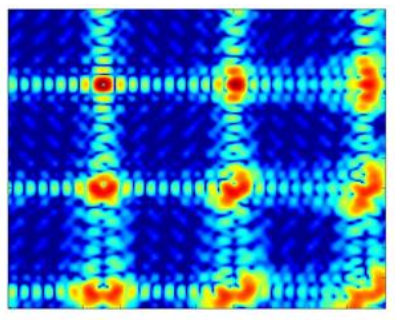

$n=1.03$

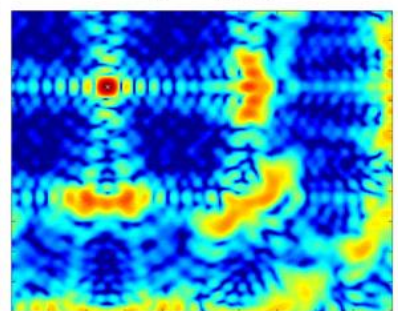

$n=1.09$

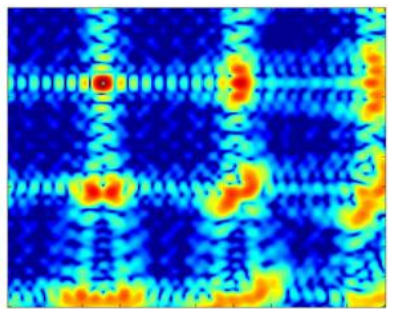

$n=1.05$

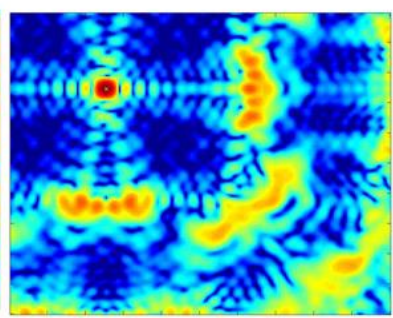

$n=1.11$

Fig. 4. Perturbing a regular array layout using a conformal power map (with aperture constant) results quickly in the smearing of grating lobes present in the far-field directivity profile. The top left image has 8 grating lobes (and the main lobe in the top left 
of that image). Only the main lobe remains localized after perturbation.

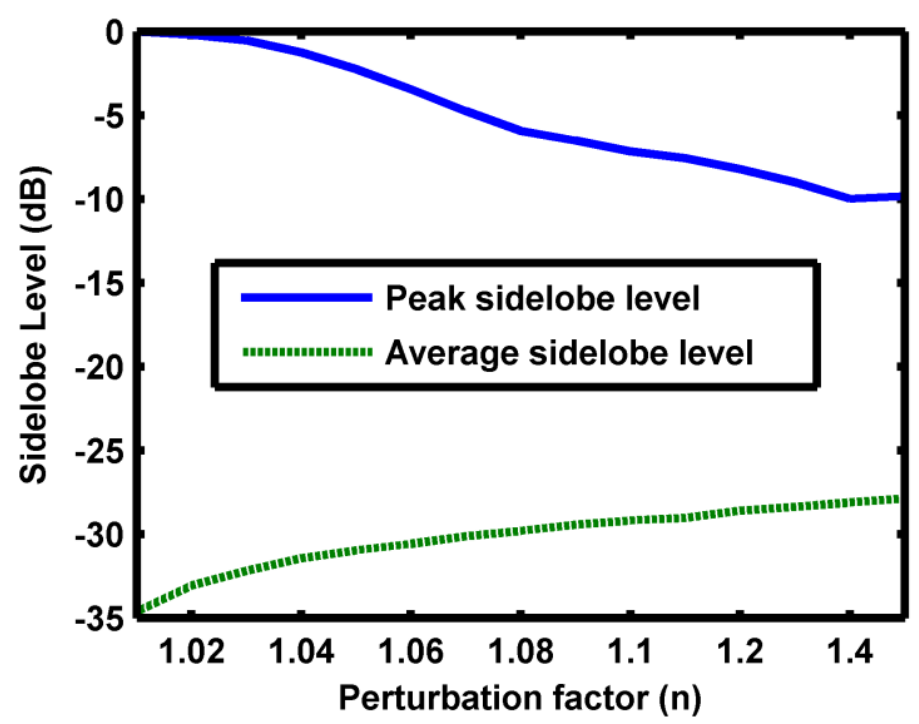

Fig. 5. Sidelobe level (normalized to the largest sidelobe at $n=1$ case) as a function of the map $z^{\wedge} n$. Increasing the power factor reduces peak sidelobe level (in grating lobes). However this results in increasing integrated sidelobe levels.

Second, for various values of $n$, the map can become degenerate - that is, separate elements in the rectangular array can occupy the same position in the mapped array. For example, for the value $n=4 / 3$, there exists a reduction in the number elements by $\sim 25 \%$. This constitutes an efficient and predictable way of reducing the number of elements in an array, in contrast to thinning through optimization techniques [11]. There is a choice to be made on how to physically create the new array design. If the position of the elements is mapped, then that only defines the new position, not the size of the new element. It is possible, of course, to map the element size as well, which creates non-uniform element sizes as a function of position. Furthermore, in some maps, the new element position is sufficiently close to another to allow for a combined element to be used; how one chooses to design the sizes of individual elements is not discussed in detail here - this work is concerned primarily with the application of conformal mapping techniques to simply map array element positions.

Fig. 6 illustrates a few examples within the range $1<|n|<3$. Once a design is chosen, it is trivial to scale the design to a required aperture size through multiplication by a constant $a$, as the conformal map we study $f(a z)=(a z)^{n}=a^{n} f(z)$ is not structurally modified by the constant scaling factor $a$. For the example configurations shown in Fig. 6, the array element layout for $n=4 / 3$ (Fig. 6(c)) is interesting for application as a 2D sparse array, and is the focus of the remainder of this article. Using this configuration, Fig. 7 demonstrates how the reduction (via smearing) of grating lobes is maintained even if we continue to increase the aperture (i.e. element spacing $>\lambda / 2$ ), which renders manufacture easier. As we increase the scaling factor $a$, the aperture and inter-element spacing increases. In a rectangular array, grating lobes would appear or increase in number due to the periodic nature of these lobes, but this conformally mapped array pattern does not have this periodicity - the beam pattern is invariant under scaling. 

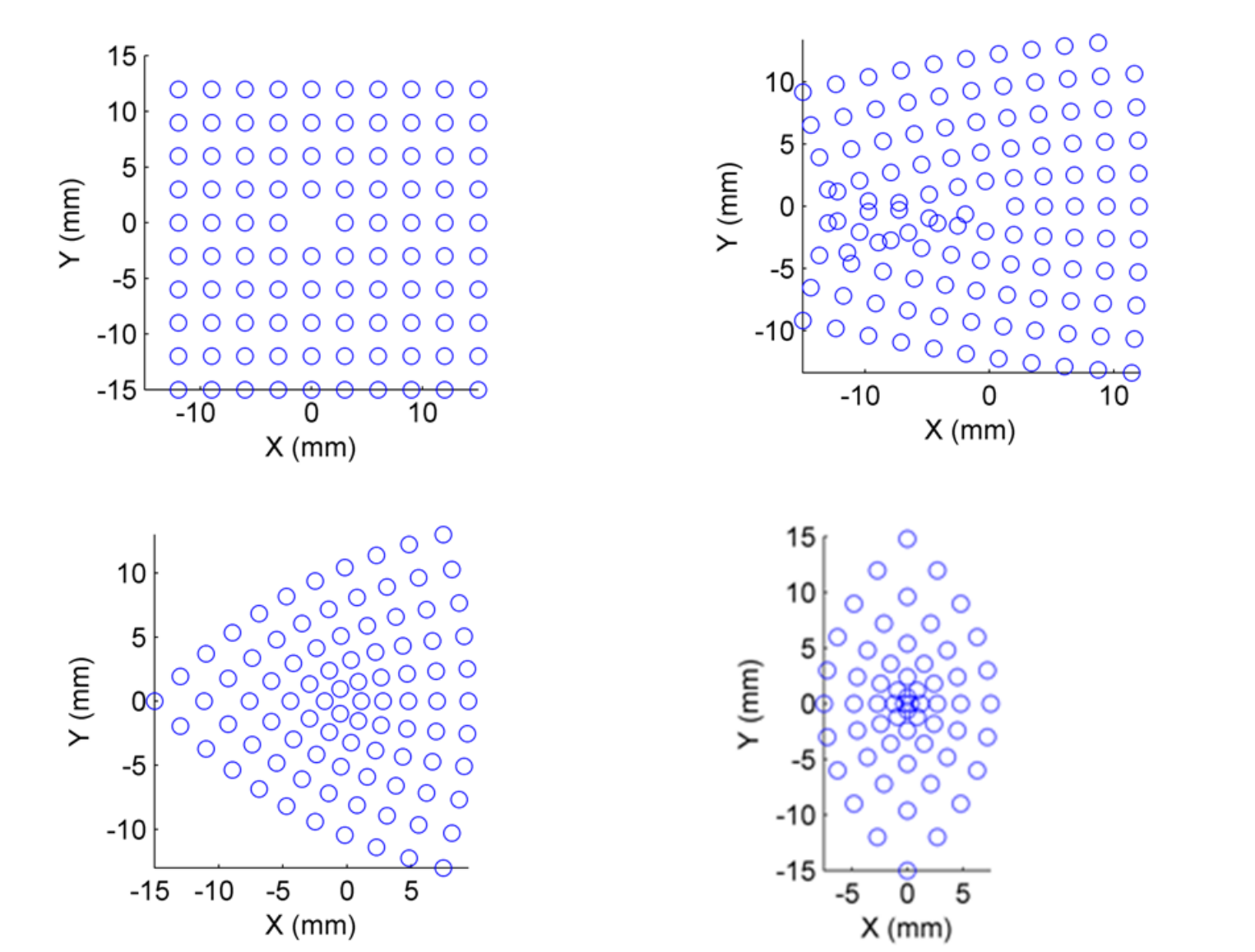

Fig. 6. Conformal power transformation of a rectangular layout where (a) $n=1$, (b) $n=1.1$, (c) $n=1.33$ and (d) $n=2$.

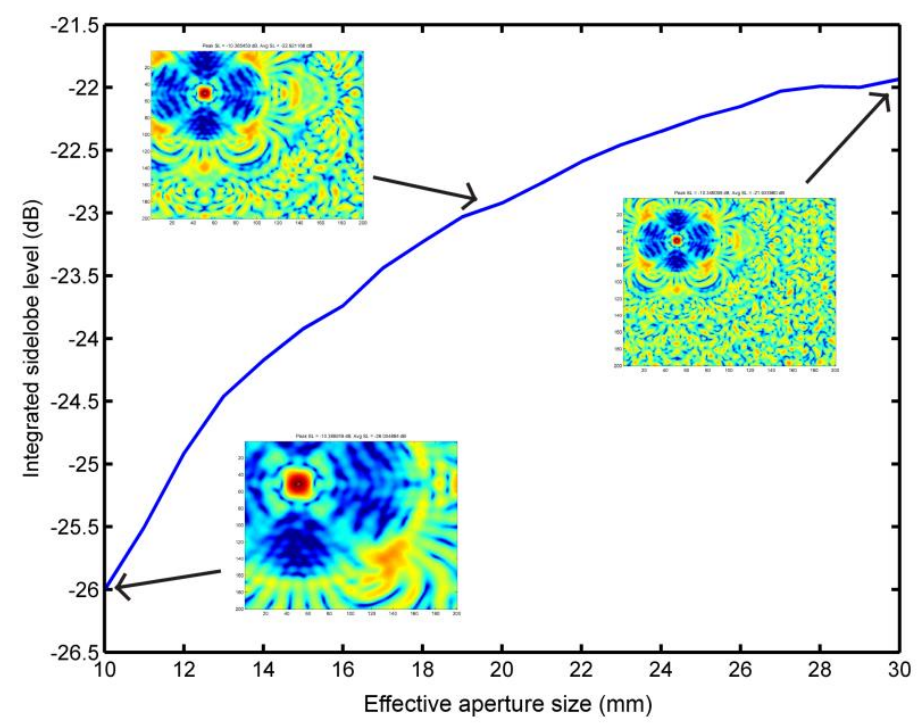

Fig. 7. Effect of scaling factor $a$ on the array imaging performance for a conformal map $\left(z^{4 / 3}\right) 2 D$ array. The scaling factor $a$ is directly proportional to the effective aperture of the array, in that the increasing pattern size evidently increases aperture. The beam pattern is invariant under change of $a$. This demonstrates that this pattern can be scaled up (i.e. magnified in size and increasing the aperture concomitantly) without introducing new lobes, despite increased element separation.

\section{Comparision analysis of a selected conformal map design with other array devices using acoustic field modelling}

Array beam-pattern response was predicted using Huygens'-principle modelling software. Fig. 8 presents the acoustic field predictions from a classical rectangular array, a random array (with no discernable structure), and $4 / 3^{\text {rd }}$ power conformal mapped 
sparse array designs. The simulation procedure is as follows.

The acoustic field is simulated using two 2D cross-sections through a 3D acoustic field: (a) L'Ambert azimuthal equiareal projection of a sphere of radius equal to the focal point distance, and (b) flat surface cross-section along XZ plane. The simulation takes into account directivity pattern of individual elements (elements are not omnidirectional). From there, side lobe aperture and main lobe width is estimated.

First, a rectangular-grid aperture (RECT) probe is selected with 121 elements distributed over circular aperture. Then, a CMAP layout probe is designed to match RECT main lobe width. This results in a probe design with lower number of active elements, and significantly lower side lobe amplitude. Finally, a completely random element location probe (RND) is designed again, using twofold condition: firstly, the number of active elements in the random probe is the same as in the CMAP probe, and secondly, the aperture is adjusted to match the CMAP and RECT main lobe width. The results are collated in Table 1. The images of the beam shape are depicted in Fig. 8. Please note that all of the above probes utilize a circular overall aperture; this is to make the beam shape near circular symmetric. Only the layout of the elements inside the aperture changes. It is clear that the CMAP layout combines the best features of the RECT and RND layouts: lowered element count and improved image contrast. It is true that the random layout smears out grating lobes; however, but it also can randomly produce high side-lobe level, depending on the dominant wavelength of operation. CMAP, being more predictably aperiodic, smears the grating lobes more uniformly. This effect is particularly important for low element count probes.

Table 1. Comparison of designs for RECT, CMAP and RND probe layouts

\begin{tabular}{|c|c|c|c|c|}
\hline Layout type & Active aperture & Element count & Beam width & Side lobe amplitude \\
\hline Unit: & $\varnothing \lambda$ & {$[-]$} & $\lambda$ & $\mathrm{dB}$ from peak \\
\hline Rectangular grid (rect) & 59.8 & 121 & 2.71 & -6.7 \\
\hline Uniform random (rnd) & 62.8 & 97 & 2.71 & -10.2 \\
\hline CMAP & 65.8 & 97 & 2.71 & -11.5 \\
\hline
\end{tabular}



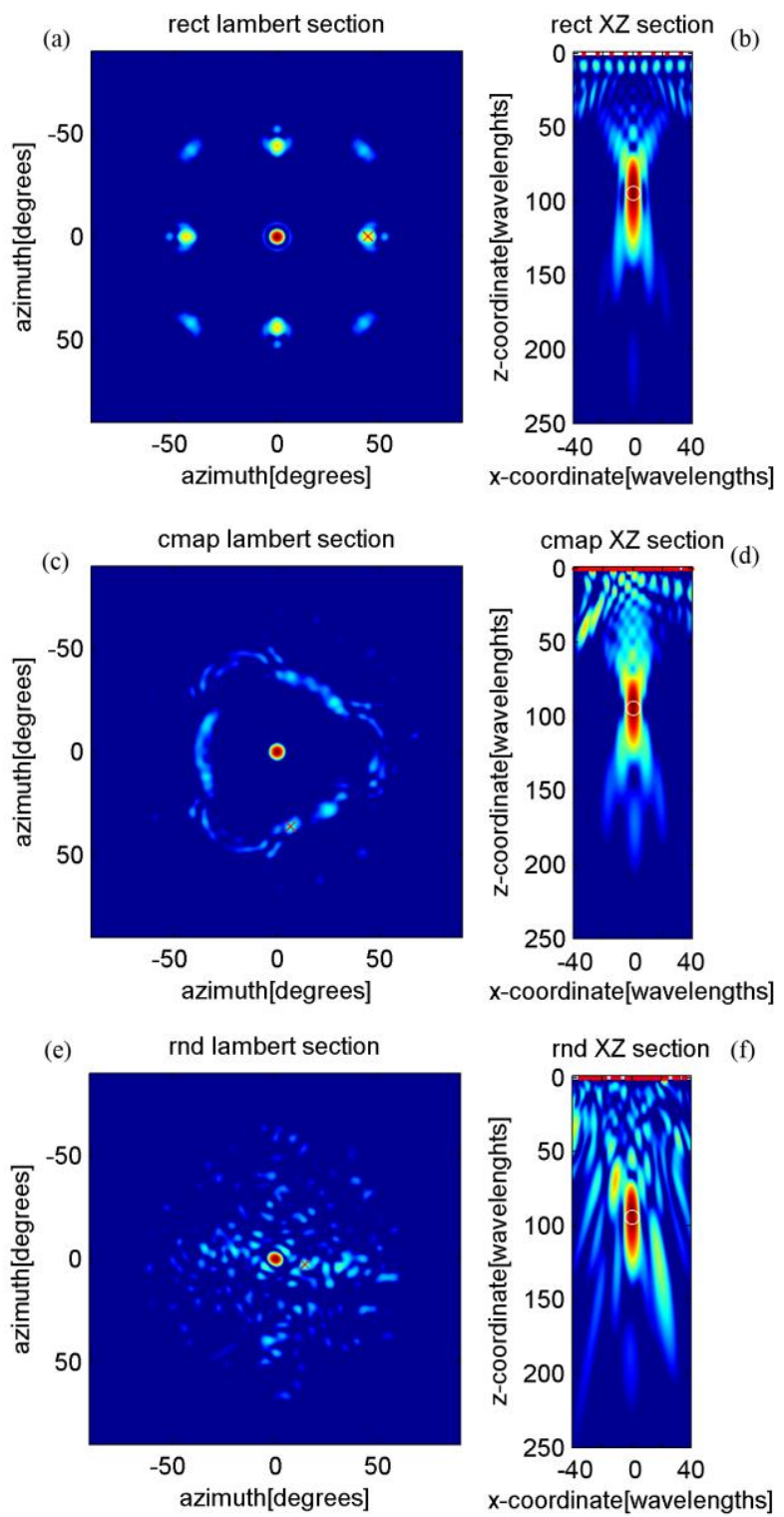

Fig. 8. Comparison of the field profiles for a rectangular, conformal-map, and random array with a focus at 100 wavelengths. Lambert sections and field profiles (in the x-z plane) of the rectangular array (a,b), conformal layout (c,d), and random array $(\mathrm{e}, \mathrm{f})$. For fixed main-lobe width, the conformal layout has a maximum lobe level of $-11.5 \mathrm{~dB}$, while the rectangular array lobe level is $-6.7 \mathrm{~dB}$. The conformal array therefore has a better contrast for fixed main-lobe width.

\section{PROTOTYPE DEVICE MANUFACTURE}

The conformal mapped sparse array configuration corresponding to $n=4 / 3$, depicted in Fig. 6(c), has been selected as the prototype array to evaluate this array design approach, with the scaling factor $a$ chosen to ensure a convenient aperture size for manufacture. In order to build the prototype conformal mapped (CMAP) array device, a 70\% volume fraction, 1-3 piezoelectric ceramic composite [13] was fabricated using PZT-5H and CIBA-GEIGY CY1301/HY1301 epoxy (Huntsman, Duxford,

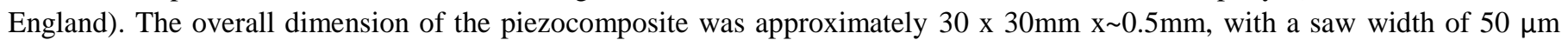
and saw pitch of $360 \mu \mathrm{m}$. This corresponds to an operating frequency of approximately $3 \mathrm{MHz}$ and was selected as this frequency has been used in recent NDE 2D array designs [3]. Gas plasma treatment was used as a precursor to physical vapour deposition of silver metallic layers to form the electrode pattern associated with the CMAP array design. The array pattern was deposited using a precision cut mask on the rear face, as shown in Fig. 9 ((a), inset). The pattern efficiently fills the 30x30mm piezocomposite, thus defining the aperture, although the non-rectangular shape means the aperture is not fully populated. A monolithic layer on the front face was also deposited to be used as the array electrical ground. Two wrap-round tags are also visible in Fig. 9 ((a), inset) and these provide an electrical connection to the ground face of the array. 
Micro-coaxial cables (132 core, $50 \Omega, 110 \mathrm{pF} / \mathrm{m}$ cable from Prosonic Ltd, South Korea) were soldered by hand onto the electroded piezoelectric composite layer. The inner conducting core of the cable is tinned to enable easy soldering of cable. Also, the individual shield wires in each micro-coax cable in the bundle (near both the cable ends) are exposed, to facilitate attaching ground connections close to the device, improving SNR. To improve the array transducer bandwidth, a light backing (approx. 2MRayl) was used to damp the device response at the rear face of the device. The backing was constructed using a syntactic foam material filled with glass spheres and epoxy resin as polymeric binder (Acoustic Polymers Ltd, Gloucestershire, UK). In addition, a $12 \mu \mathrm{m}$ protective layer made of nano-silica filled epoxy polymer (Masterbond Inc.) was used at the front face. Finally, the array was inserted carefully into an aluminum casing and potted with polyurethane as a sealant.

Individual testing of each element in the finished CMAP array showed variation in sensitivity across the array. This attribute could be due to one, or a combination, of variations in the solder attachment at the back of each element or the loading (i.e. glass spheres and cables) at the back face from the syntactic foam backing medium used. Also, there were slight variations in the polyurethane mixture used as a sealant resulting in an inconsistent performance while setting. Notwithstanding these manufacturing issues, the prototype CMAP array was considered suitable for evaluation in this feasibility study.

\section{EXPERIMENTAL RESULTS}

To characterize the CMAP array both electrical impedance and laser vibrometry measurements were acquired. The operational impedance characteristic of the CMAP 2D array substrate was measured using a HP Impedance Analyser (HP4194A, Agilent, South Queensferry, Scotland). Fig. 9(a) details the electrical impedance characteristic of the composite substrate (temporarily silvered fully on both sides) and illustrates clearly the presence of thickness mode electrical resonance at approximately $3 \mathrm{MHz}$. . Fig. 9(b) illustrates the surface displacement characteristic of the array when only one array element is excited. The array element displacement was measured at $6 \mathrm{~nm}$ using a scanning laser vibrometer (PSV-300-F, Polytec; Waldbronn, Germany) and importantly, the mechanical cross-talk across the array aperture is $20 \mathrm{~dB}$ down on the peak displacement of the active array element.
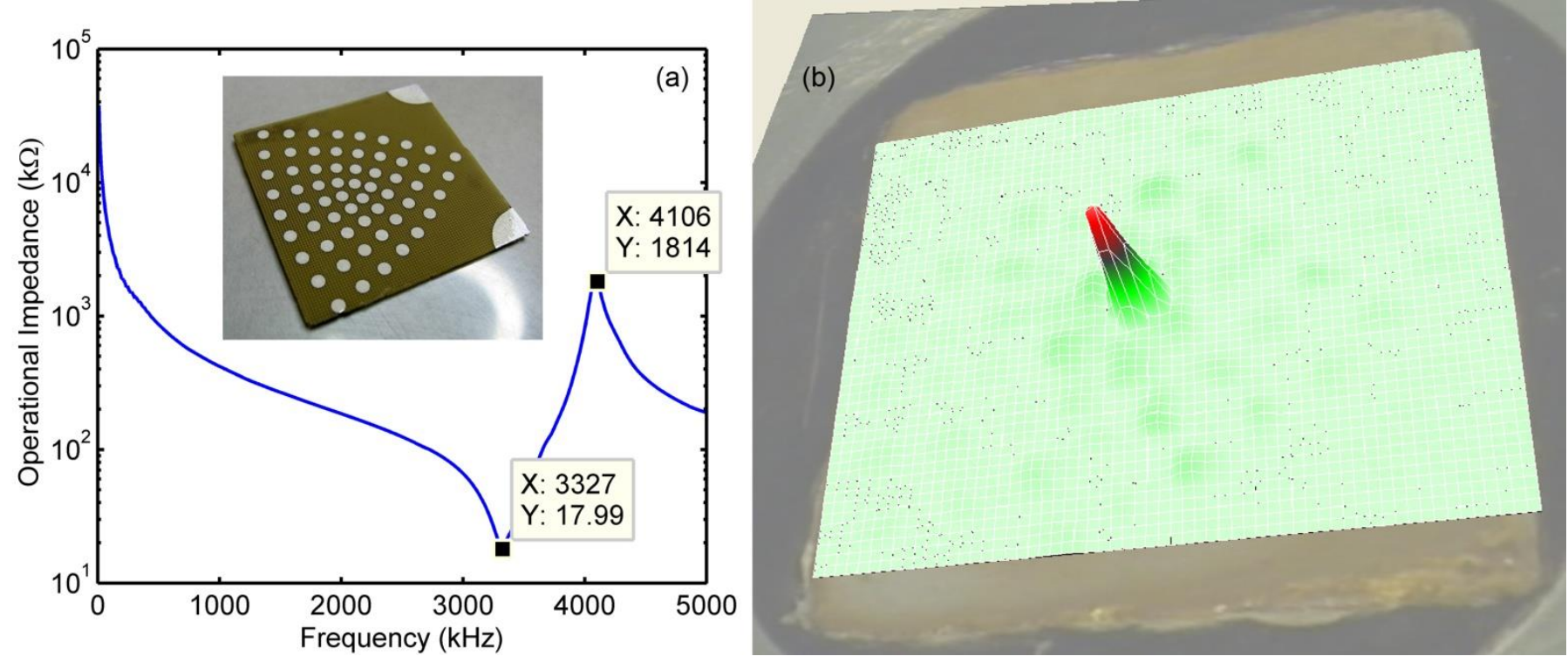

Fig. 9. (a) Electrical impedance characteristics of the 1-3 piezocomposite microstructure used in prototype CMAP construction. (inset) Silver electrode configuration applied to a 1-3 piezocomposite substrate to produce a 60element CMAP prototype array: rear face of the substrate is shown. (b) Laser vibrometry scan of the transducer, with one element being driven at $3.3 \mathrm{MHz}$, showing low levels of mechanical cross-talk across the array aperture. This example has an amplitude of $\sim 100 \mathrm{~nm}$.

To characterize the probe pulse-echo performance, it was placed on an $80 \mathrm{~mm}$ stainless steel block with back-drilled holes of $2 \mathrm{~mm}$ in diameter, and $10 \mathrm{~mm}$ depth. A Dynaray phased array controller (Zetec, Canada) was used to drive the CMAP array, with array elements excited using a 50ns, $80 \mathrm{~V}$ pulse, and the echo from the back wall was collected on all elements separately. An example pulse-echo response is shown on Fig. 10(a). Full Matrix Capture (FMC), a specific data acquisition process where all individual transmit-receive time domain signals within an array are captured [14], has been acquired using the prototype CMAP array. The 3D total focussing method was applied in post-processing the FMC data [7] and the volumetric scan of the steel test 
specimen is presented in Fig. 10(b). The responses from individual elements were not equalized, hence the 'rough' appearance of the back wall in Fig. 10(b). Notwithstanding, the two holes are clearly visible in the image, and there are neither grating lobes nor ghost images of the reflectors.
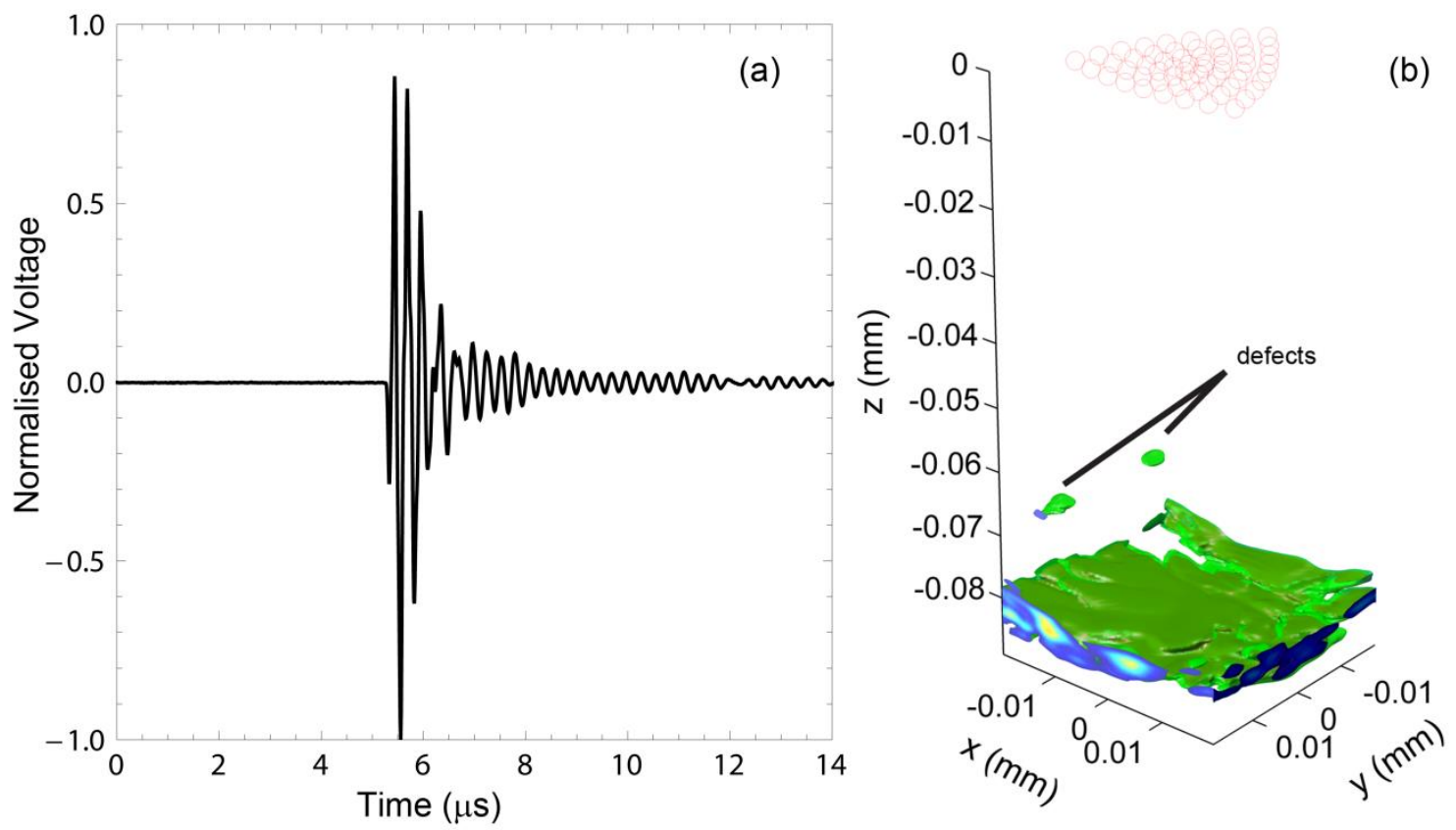

Fig. 10. Experimental data collected by CMAP array on a steel test block $80 \mathrm{~mm}$ thick with two $10 \mathrm{~mm}$-deep drilled holes. (a) Pulse-echo response of an array element (with normalised voltage. (b) 3D image from the Total Focussing Method showing the back wall at $80 \mathrm{~mm}$ and two holes.

\section{CONCLUDING REMARKS AND SUGGESTIONS FOR FURTHER DEVELOPMENT}

A conformal map based method to design sparse 2D array configurations has been presented. The investigations indicate that such an approach could be employed to efficiently break the periodicity in an array lattice, which allows the array structure to be scaled without introducing undesirable grating lobes. In the adopted design methodology, the array element position only was selected to undergo the mapping process. Mapping array element shape in conjunction with its spatial coordinates could generate interesting apodisation schemes and could be studied separately.

A prototype $4 / 3^{\text {rd }}$ power conformal transformation based layout device was manufactured and the preliminary results are encouraging. While such sparse array design permits the development of a reduced element count larger aperture, high frequency, 2D array suitable for imaging application, there is a limit to how large an array could be built using standard array manufacture and assembly procedure. For example, at high frequencies the thickness mode piezoelectric layer is very thin and the conventional array assembly stages (including electroding, wiring, and bonding front/back face layer) could prove problematic, especially for producing extremely large radiating front face. A compromise between array imaging performance (in terms of beam spread, sidelobe level, and operating frequency) and manufacturing complexity is necessary while designing such high frequency, sparse array devices to suit a particular application.

The potential for a conformal map of the form $w=z^{n}$ to create sparse 2D arrays has been demonstrated and greater image contrast can be achieved when compared to conventional densely populated array configurations. Transformations of this type (i.e. conformal mapping) provide an interesting addition to array layout design that combines symmetry breaking in an analytic context, and other maps could potentially yield even greater performance.

\section{ACKNOWLEDGMENT}

The authors would like to thank EPSRC (JCJ - EP/H02848X/1) \& RCNDE for funding the project, Messrs. T. McCunnie and G. Smillie for their help during fabricating of the prototype CMAP array, Miss A. McGilp for obtaining data, and Dr. A. Velichko and Prof. P. D. Wilcox, at Bristol University, for their input during this work as part of a collaborative EPSRC project (EP/F004680/1). 


\section{REFERENCES}

[1] J. Krautkrämer, H. Krautkrämer, Ultrasonic Testing of Materials, $4^{\text {th }}$ ed. Germany, Springer-Verlag, Berlin Heidelberg, 1990.

[2] B. W. Drinkwater, P. D. Wilcox, "Ultrasonic arrays for non-destructive evaluation: A review", NDT \& E International, vol. 39, no. 7, pp. 525-541, Oct. 2006.

[3] Velichko, A. and Wilcox, P. D., "Strategies For UltrasoundTwo-Dimensional Arrays", Review of Progress in QNDE, eds. Chimenti, D. E., Thompson, D. O., vol. 29(A), pp. 887-894, Mar. 2010.

[4] O. Martinez-Grauller, C.J. Martin, G. Godoy, L.G. Ullate, '2D array design based on Fermat spiral for ultrasound imaging', Ultrasonics, vol. 50, no. 2 , pp. 280-289, Feb. 2010

[5] P. Wilcox, A. Velichko, "Defect characterization using two-dimensional arrays", Review of Progress in QNDE, vols. 30A AND 30B, pp. 835-842, 2011

[6] O. Martinez, M. Akhnak, L.G. Ullatem, F.M. de Espinosa, "A small 2D ultrasonic array for NDT applications", $N D T$ \& $E$ International, vol. 36, no. 1, pp. 57-63, Jan 2003

[7] A. Tweedie, R.L. O'Leary, G. Harvey, A. Gachagan, C. Holmes, P.D. Wilcox, B.W. Drinkwater, "Total focussing method for volumetric Imaging in immersion non destructive evaluation", Proc. IEEE Ultrasonics Symposium, pp. 1017-1020, Oct. 2007

[8] E. W. Weisstein, "Conformal Mapping", From MathWorld--A Wolfram Resource, http://mathworld.wolfram.com/ConformalMapping.html, July 2012

[9] NASA Web Resource, "Beginner's Guide to Aerodynamics", http://www.grc.nasa.gov/WWW/K-12/airplane/map.html, July 2012

[10] U. Leonhardt, "Optical Conformal Mapping", Science, vol. 312, no. 23, pp. 1777-1780, June 2006.

[11] A. Trucco, "Thinning and Weighting of Large Planar Arrays by Simulated Annealing", IEEE Trans. Ultrason. Ferroelectr. Freq. Control, vol. 46, no.2, pp. 347-355, Mar. 1999.

[12] S. Holm, B.Elgetun, G. Dahl, "Properties of the beampattern of weight- and layout-optimized sparse arrays", IEEE Trans. Ultrason. Ferroelectr. Freq. Control, vol. 44, no. 5, pp. 983-991,Sept, 1997

[13] W.A. Smith, B. A. Auld, 'Modeling 1-3 composite piezoelectrics - thickness-mode oscillations ', IEEE Trans. Ultrason. Ferroelectr. Freq. Control, vol. 38, no. 1, pp. 40-47, Jan 1991

[14] C. Holmes, B. W. Drinkwater, and P. D. Wilcox, "Advanced post-processing for scanned ultrasonic arrays: Application to defect detection and classification in non-destructive evaluation", Ultrasonics, vol. 48, pp. 636-642, Nov 2008. 